\title{
Improving post-EVAR surveillance with a smart stent-graft
}

\author{
Isa C.T. Santos ${ }^{1}$, Alexandra T. Sepulveda ${ }^{2}$ Júlio C. Viana ${ }^{2}$, António J. \\ Pontes $^{2}$, Brian L. Wardle ${ }^{3}$, S. M. Sampaio ${ }^{4}$, R. Roncon-Albuquerque ${ }^{4}$, João \\ Manuel R.S. Tavares ${ }^{1}$ and L.A. Rocha ${ }^{2}$ \\ ${ }^{1}$ Faculty of Engineering, University of Porto / Institute of Mechanical Engineering and \\ Industrial Management, Porto, Portugal \\ ${ }^{2}$ Institute for Polymers and Composites/I3N, University of Minho, Portugal \\ ${ }^{3}$ Technology Laboratory for Advanced Materials and Structures, Department of Aeronautics \\ and Astronautics, Massachusetts Institute of Technology, Cambridge, MA 02139, USA \\ ${ }^{4}$ Vascular Surgery Hospital S. João, Faculdade de Medicina, Universidade do Porto, Portugal
}

\begin{abstract}
Abdominal aortic aneurysms (AAA or triple A's) are indolent and deadly diseases. Their treatment options involve either an invasive procedure or a minimally invasive one. When the minimally invasive procedure, endovascular aneurysm repair (EVAR), was introduced, it revolutionized the treatment of AAA's due to advantages such as shortened hospital stays and reduced costs. As EVAR requires periodic imaging exams, questions are nowadays being raised regarding the procedure's long-term cost-benefit relation. In order to reduce followup costs, new technological solutions are being pursued, namely EVAR stentgrafts with sensing capabilities. In this chapter, the suitability of aneurysm sac pressure measurement for EVAR surveillance is evaluated using an AAA computer model. In addition the design drivers underlying EVAR stent-grafts are reviewed and the development of a new flexible pressure sensor integrated into a stent-graft is described.
\end{abstract}

\section{Introduction}

In 1948, Albert Einstein was diagnosed with an abdominal aortic aneurysm (AAA), i.e., his aorta had a permanent and irreversible localized dilatation having at least a $50 \%$ increase in diameter compared with the normal one [1], Fig. 1. Like him, currently, it is estimated that more than 12 per 100000 persons-year [2] are affected by this relatively indolent but serious condition.

In an attempt to reinforce the aortic wall and delay the inevitable rupture that took Einstein's life in 1955, Dr. Rudolph Nissen wrapped the visible anterior portion of the aneurysm with polyethene cellophane. Nowadays, two more effective treatments are available: open surgery and endovascular aneurysm repair (EVAR). 
While the first is an invasive procedure, the second is a minimally invasive one whose main advantages are decreased blood loss, less early morbidity and mortality, and shorter hospitalization [2].

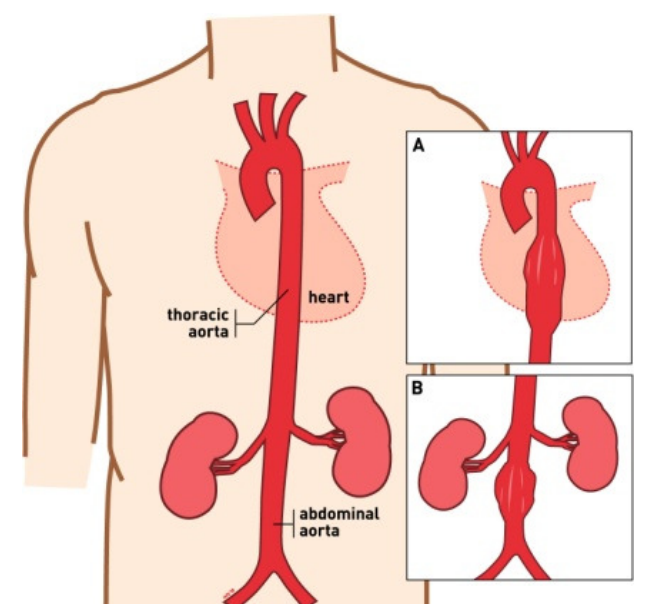

Fig. 1. Representation of a normal aorta, (A) a thoracic aortic aneurysm (TAA), and (B) an abdominal aortic aneurysm (AAA).

When EVAR was introduced, it revolutionized the treatment of aortic aneurysms. However, and in spite of major advances in EVAR techniques, adverse reactions still occur [3] and lifelong surveillance is recommended [4]. Due to these complications, currently, questions are being raised regarding the follow-up costs [5] and alternative approaches, such as a smart stent-graft, are being pursued.

A stent-graft is an endoprosthesis composed of a metallic scaffold and a polymeric covering membrane. Its use and the technological background for the development of a new device with sensing capabilities are described in this chapter.

After describing the current treatment options for aortic aneurysms, stent-graft complications are presented as well as their surveillance techniques. Following, the devices to measure pressure inside the aneurysm sac are described. The future trends for stent-graft's are also addressed highlighting the features of a smart stent-graft. A model of an AAA is presented along with a description of the development of a flexible pressure sensor.

\section{Endovascular Aneurism Repair}

Since the early 1950's, aneurysms' standard treatment has consisted of an open surgery (done under general anesthesia) and the replacement of the diseased segment of the aorta by a synthetic prosthetic graft [6], Fig. 2. In spite of its invasiveness and the fact of being limited to fit patients, this treatment is still a current 
practice and less invasive techniques, namely total laparoscopy and assisted laparoscopy, are being studied to minimize its disadvantages [7].

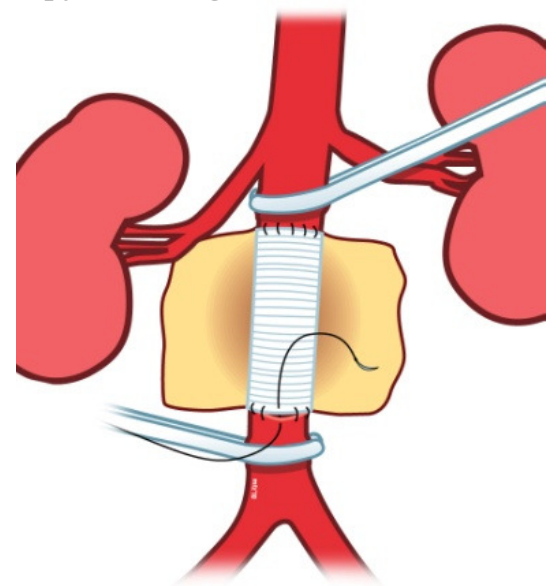

Fig. 2. Conventional treatment of AAA's, open surgery to insert a synthetic graft.

In the beginning of the 1990's, Volodos (in Ukraine) and Parodi (in Argentina) demonstrated that endovascular aneurysm repair (EVAR) was a safe and feasible practice [8]. This surgical procedure is done percutaneously and it is minimally invasive, Fig. 3. Typically, a small incision is made in each groin to expose the femoral arteries. Then, with the aid of catheters and guidewires, a stent-graft is guided to the affected artery segment allowing blood to pass without exerting pressure in the aneurysm sac and, thus, preventing wall rupture.
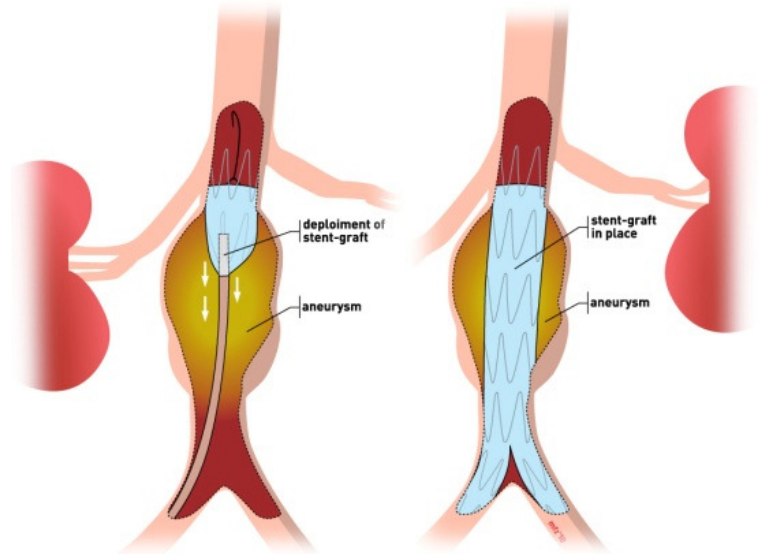

Fig. 3. Endovascular aneurysm repair (EVAR), stent-graft deployment sequence.

The first procedures resulted in several complications, some due to the inherent learning process, while others were due to the devices' inefficiency. With time, many of the problems have been solved due to the accumulation of experience and 
the introduction of better devices but, nevertheless, some problems still occur. These complications can be classified as early or late and further detailed as systemic or related with the delivery, deployment, or the device itself [3]. Table 1 presents some of EVAR's problems involving stent-grafts.

Table 1. Complications involving stent-grafts (adapted from [3]).

\begin{tabular}{ll}
\hline Early complications & Late complications \\
\hline Graft kink & Graft migration \\
Endoleaks & Neck dilatation \\
Graft explantation & Endoleaks \\
Structural failure & Structural failure: component separation, fa- \\
Graft infection & bric tears, hook fractures \\
\hline
\end{tabular}

The current surveillance protocol involves imaging exams, namely ultrasound and computed tomography angiography (CTA), at 1, 6, and 12 months after the procedure, and thereafter, on an annual basis [9].

Device migration and stent fractures or other indication of device fatigue are clear in plain abdominal radiography. Ultrasonography, besides allowing measuring the aneurysm sac, is effective in the detection of endoleaks but, even with enhanced sensitivity obtained with the use of contrast agents, requires a skilled technician to interpret the exams. CTA, MRI (magnetic resonance imaging) and MRA (magnetic resonance angiography) are sensitive tools to detect endoleaks but cannot be repeated often due to radiation and/or the use of nephrotoxic contrast agents. Furthermore, these exams are considered time consuming and expensive.

\section{Measurement of Aneurism Sac Pressure}

None of the medical imaging exams presently used provides measures of the pressure inside the aneurysm sac. This information is important because can be evidence of low-flow endoleaks or endotension [10].

Published data describe the use of catheters to measure pressure in the residual aneurysm sac [11]. However, although these methods provide precise measurements [4], they are invasive and bear multiple risks.

An alternative method for the measurement of the aneurysm sac's pressure is the implant of remote pressure transducers during EVAR. This solution is advantageous since measurements can be done when needed (hourly, weekly, etc.) in the patient's home or office instead of a hospital once or twice a year. Another important feature is the possibility to measure both the mean pressure and the pulsatile pressure without increasing risks for the patients. Following, the three telemetric pressure sensors currently available will be addressed. 


\section{Impressure AAA sac pressure transducer}

In 2003, the Impressure AAA Sac Pressure Transducer or RemonAAA from Israeli Remon Medical Technologies was the first permanently implantable, ultrasound-activated remote pressure transducer to measure intrasac pressure after EVAR [12].

The transducer, hand sewn to the outside of a stent-graft, contains a piezoelectric membrane that energizes a capacitor when actuated by ultrasound waves from a hand-held probe. Once charged, the aneurysm sac pressure is measured followed by the generation of an acoustic signal that is relayed to the hand-held probe. The probe then converts the acoustic signal to a pressure waveform that is presented on a computer screen.

In spite of ultrasound being safe and widely used for medical imaging, the measurement requires the use of an ultrasonic gel and direct contact between the skin and the transducer. Another drawback of this sensor is the impossibility of ultrasound to travel through air or bone, which may lead to difficulties communicating with the aneurysm sac.

The ImPressure sensor is the smallest $(3 \mathrm{~mm} \times 9 \mathrm{~mm} \times 1.5 \mathrm{~mm})$ of the three sensors described here, is the least radiopaque but still visible. As the sensor is sewn to the stent-graft, the fixation location must be carefully chosen in such a way that the sensor will measure the pressure inside the excluded aneurysm sac without being pushed against the aneurysm wall.

In October 2006, Remon Medical Technologies announced the first European implant of the sensor.

\section{EndoSure wireless pressure sensor}

The EndoSure Wireless Pressure Sensor (CardioMems, Inc., USA) is made of two coils of copper wire within a fused silica matrix with a pressure sensitive surface. Passive telemetry is used for the signal transfer between the external device and the implant. Through inductive coupling, changes on the internal LC network (capacitor plus inductor) resonance frequency are detected on the external coil. The resonance frequency is related to the ambient pressure in which the sensor is located, and specially-designed software transforms the frequency shift between systolic and diastolic pressures into a wave form and pressure reading [13].

As the measurements are acquired via RF, there is no need for contact with the patient's skin or even the removal of the clothes, potentially allowing daily/weekly sampling at home.

The EndoSure sensor is deployed through its own delivery catheter (diameter $14 \mathrm{Fr} / 4.7 \mathrm{~mm}$ ) during the EVAR procedure and has radiopaque markers to clearly define its location within the aneurysm sac.

The EndoSure sensor is cleared by the FDA for the measuring of intrasac pressure during endovascular abdominal aortic aneurysm repair and during endovascu- 
lar thoracic aneurysm repair but is not yet approved for use in chronic surveillance.

\section{TPS telemetric pressure sensor}

The TPS Telemetric Pressure Sensor was developed by the Helmholtz-Institute for Biomedical Engineering, RWTH Aachen in cooperation with the Institute of Materials in Electrical Engineering, RWTH Aachen [13-14].

The TPS sensor consists of an implantable sensor capsule and an external readout station. The capsule comprises a capacitive absolute pressure sensor and an in-capsule signal-processing microchip including an inductive telemetry unit. The measured data is fully preprocessed to a digital data stream in the implant, so that errors during the transmission or from interferences between the sensor and the external readout station can be minimized. Moreover, the additional integrated temperature sensor allows errors caused by temperature variations to be noted and numerically corrected. In keeping with the requirements of medical implants, the pressure capsule has a two-layer encapsulation: a biocompatible, capsule-shaped silicone form and a Parylene-C layer creating an enclosed covering and ensuring the required stiffness.

The TPS sensor has fixation holes in both ends allowing to be sutured to the outer wall of stent-graft or be introduced separately.

This device has only been tested in an in vitro model but the results demonstrate that this is a promising technology [15]. Nonetheless, further clinical studies are required to evaluate the TPS Telemetric Pressure Sensor's durability and accuracy.

\section{EVAR cost benefit}

Comparing EVAR with conventional surgery, the first is preferable due to the fact of being less stressful and reducing significantly systemic complications [16], as well as having lower costs of inpatient stay and less or no need for intensive care facilities during recovery $[6,17]$. While a number of early studies appeared to support this claim, nowadays, data shows otherwise [16]. Shorter stays at intensive care units and the hospital, reduced use of blood, fewer laboratory studies and fewer resources lead to cost savings, but later, additional costs exist for EVAR due to surveillance procedures.

The durability of open surgery, established with long-term follow-up studies, is excellent [16], so good that there is little or no requirement for long-term surveillance. Hayter [17] compared both hospital and follow-up costs of patients who had undergone EVAR or open surgery and concluded that EVAR costs were higher. One of the justifications presented was the endograft's high price. 
When EVAR was introduced, it was thought to be more economical than open surgery because the price of the first stent-grafts was lower and the surveillance costs were not included in the analysis. Nowadays, EVAR can be considered costeffective only for very elderly patients or those with a reduced life expectancy and doubtful for young patients, those who would benefit more from the short hospital stay and early return to full activity offered by open surgery.

Considering the longer life expectancies and the rising public expectations for quality of life, EVAR is an attractive treatment. However, its cost-benefit relation can be jeopardized by the requirement of long-term surveillance. In order to reduce and even eliminate these exams, new surveillance technologies are being investigated and the most promising technique identified thus far is remote pressure sensing [9].

The authors believe that including sensing capabilities in a stent-graft will benefit EVAR's future. Yet, that may not be enough. Preliminary results of a recent survey regarding the ideal features of a stent-graft show that attention should be given to the devices adaptability and delivery profile.

\section{Future Trends for Stent-grafts}

Innovations on mechanisms for pressure detection within the aneurysm sac are being developed concurrent with novel architectures and materials for the construction of the stent-graft. Two original proposals that indicate future alternatives for stent-graft design are presented next.

\section{Origami stent-graft}

In 2006, Kuribayashi [18] described the design, the manufacture and the properties of an origami stent-graft. The new device received this designation because the paper folding patterns used in the Japanese art of origami was employed to fold it.

This prosthesis is made from a sheet that is folded dividing a cylindrical tube into a series of identical elements with hill and valley folds. The folding pattern used is responsible for the decrease and increase of both the diameter and the length when the device is folded and deployed, respectively. In addition, the folded configuration of each element makes the stent-graft flexible.

Unlike other devices, the origami stent-graft is made of a single component. A nickel titanium alloy is used that not only is biocompatible, but also has a shape memory effect that is used for the deployment of the device. Its main disadvantage is the price; the origami stent-graft is made using a foil that requires complex rolling and annealing methods to be produced. 


\section{Rigberg's stent-graft}

The major determinant of the stent-graft's diameter when folded is the graft, not the stent itself. Thus, in order to reduce the pre-deployment diameter and the size of the delivery components, Rigberg presented in 2009 a feasibility study for a novel aortic stent-graft material [19]. In his work, the author proposes the replacement of currently used graft materials by a nickel titanium alloy (NiTi) thinfilm. This material presents several advantages, such as biocompatibility, superelastic qualities, shape memory properties, and a tensile strength of greater than 500 $\mathrm{MPa}$. This last feature is of major importance since it enables the development of thinner devices with the same, or even higher, mechanical resistance. Moreover, the cost of thin-film NiTi is expected to be similar to the cost of ePTFE. Nonetheless, further studies regarding thrombogenicity, resistance to infection, and permeability are still required. Some design issues, such as the attachment of the stent to the graft or complex shapes, also need to be more deeply studied.

\section{Smart stent-graft}

A smart stent-graft can be defined as a stent-graft with some in-device mechanism to perform a given function with communication capabilities to an external element.

Although there is still no commercial device available, a smart stent-graft could be decomposed in three elements: a stent-graft, a sensing element and a display. The stent-graft, besides shielding the aneurysm from the blood pressure, has builtin sensing elements that are able to gather information concerning the patient's health and/or the prosthesis performance. The information gathered is then sent to an external element - a display - and can be used to diagnose the patient's or in the comprehension of the aneurysm's sac behavior after the implementation of the stent-graft.

Like a conventional stent-graft, such a device will be classified as a class III medical device and, as such, will have to be biocompatible, biostable, non-toxic, non-allergic and non- carcinogenic. Furthermore, it will have to be tolerated by the human body without causing a foreign body reaction or an inflammatory reaction.

Regarding the mechanical requisites, the device should be flexible and tough Its components should also be mechanically durable, as well as excellent corrosion resistance. For a successful protection of the blood vessel, the device should have a design as less invasive as possible in order to minimize flow resistance and pressure drops. Radial force is another relevant feature, not only for stent-grafts to stay open without being crushed with muscular activity, but also to provide a good seal and to ensure fixation.

The deployment of the device is a critical step for the procedure's success, thus, the stent-graft should have a low profile to facilitate the deployment and minimize 
lesions in the access arteries. At this stage, radiopacity is also crucial to ensure the correct positioning of the prosthesis.

From the commercial point of view, the device must be capable of being adequately sterilized and stored as an "off-the-shelf" product. A broad range of sizes is desirable since it allows the treatment of a wider array of aneurysm anatomies.

One of the key questions in the design of a smart stent-graft regards the instrumentation capabilities required. Ideally, the device should be able to detect migration and leakages and possibly also monitor any device material or structural degradation. Regarding the transmission of the measured data, the device must be able to transmit the data without any internal power. Moreover, the data cannot interfere with other implants nor be influenced by other electronic signals.

To assure patient's comfort and even reduce costs, the measurement protocol should be done during the doctor's appointment or at home and the results transmitted to the doctor's office. Regardless of where measurements are taken, the procedure should be quick, the least invasive as possible and avoid any kind of pain or even discomfort.

For a correct interpretation of the information measured, it is crucial to know the location where the data is being gathered.

\section{Abdominal Aortic Aneurysm Model}

Thus far, to measure the aneurysm sac's pressure, sensors are placed randomly and provide information regarding a single point. This data is insufficient to characterize the pressure distribution inside the aneurysm sac. Therefore, AAA models using finite elements can become a means to understand the medical condition and predict the optimal location to place the sensors.

Several studies using AAA models composed of a stent-graft, the aorta and the aneurysm sac have been presented in the literature [20-22], but they mainly studied the drag forces on the stent-graft, or the stresses on the vessel that can lead to rupture. Even though this information is important to improve existing knowledge about aneurysms, it conveys no detail about the pressure distribution, or the influence of AAA geometry on the aneurysm sac pressure.

Using computer-aided design (CAD) software, it is possible to implement parametric models to construct tridimensional (3D) models of the desired AAA in a fast and efficient manner, as in Fig. 4. For an accurate representation, the model must include the blood flow, the bifurcated stent-graft, the aorta wall motion (including the aneurysm wall) and the stagnant blood inside the aneurysm sac (essential for the pressure simulation inside the sac).

Due to the pulsatile nature of blood flow, transient simulations are often used to model the blood flow. Aneurysms can have several geometries and sizes [20,23], but AAAs present most commonly a fusiform geometry. The aorta radius ranges from 2 to $2,5 \mathrm{~cm}$ and the wall thickness presents typical values around $2-2,5 \mathrm{~mm}$ $[20,23]$. 


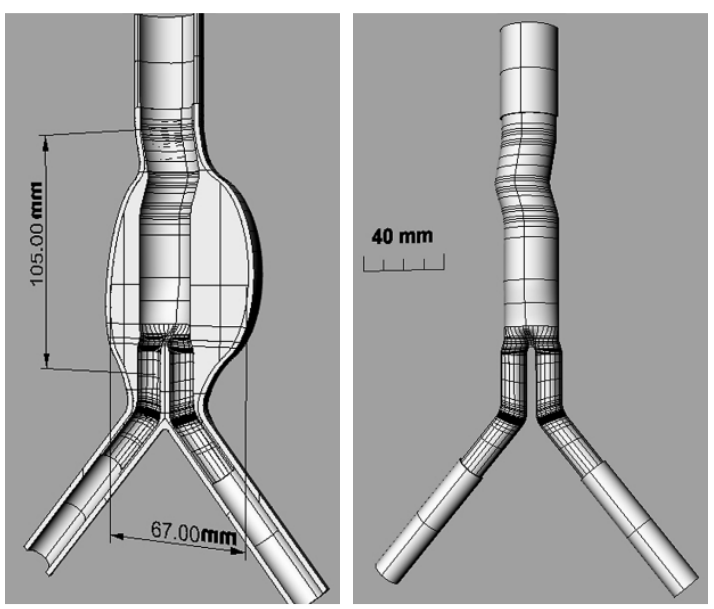

Fig. 4. 3D models of an AAA. a) mechanical model and b) fluidic model.

Fig. 5 presents the main parameters of the 3D model implemented by the authors to assess the pressure distribution inside the aneurysm sac. The ANSYS multiple code coupling (MFX) with Fluid Solid Interface (FSI) coupling between ANSYS and CFX was used to solve the model.

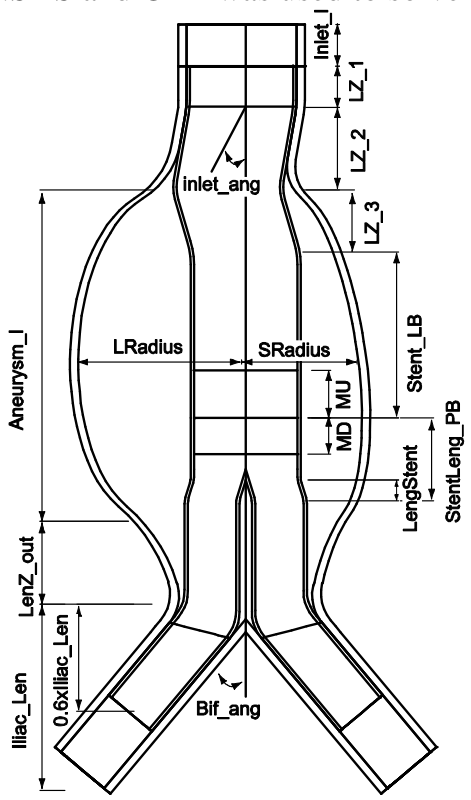

Fig. 5. Main modeling parameters of an AAA.

The blood flow is considered newtonian, laminar and incompressible; the density is equal to $1.05 \mathrm{~g} / \mathrm{cm}^{3}$ and the viscosity is $0.0035 \mathrm{~Pa} . \mathrm{s}$. 
The model uses an aorta radius and aorta wall thicknesses of $2.5 \mathrm{~cm}$ and 2.5 $\mathrm{mm}$ respectively, and an aneurysm length of $10.5 \mathrm{~cm}$ and main radius of $6.7 \mathrm{~cm}$.

The diseased AAA wall is modeled as a linear, isotropic, elastic material with a density $\rho=1.2 \mathrm{~g} / \mathrm{cm}^{3}$, a Young's Modulus $\mathrm{E}=4.6 \mathrm{MPa}$ and a Poisson's ratio of 0.49 [20].

The healthy part of the aorta (AAA neck) and iliacs are also modeled as a linear, isotropic, elastic material with a density $\rho=1.2 \mathrm{~g} / \mathrm{cm}^{3}$, a Young's Modulus E $=2 \mathrm{MPa}$ and a Poisson's ratio of 0.45 .

The stent-graft model presented uses SHELL elements, and is modeled as a linear, isotropic material with a Young's Modulus E $=10 \mathrm{MPa}$ and a Poisson's ratio of 0.3 .

The aneurysm sac, an important part of the model for the pressure analysis, is modeled as a stagnant liquid by using FLUID80 element from the ANSYS element library. This element allows the simulation of stagnant fluids in containers with no flow. Fig. 6 gives an overview of the meshed mechanical model.

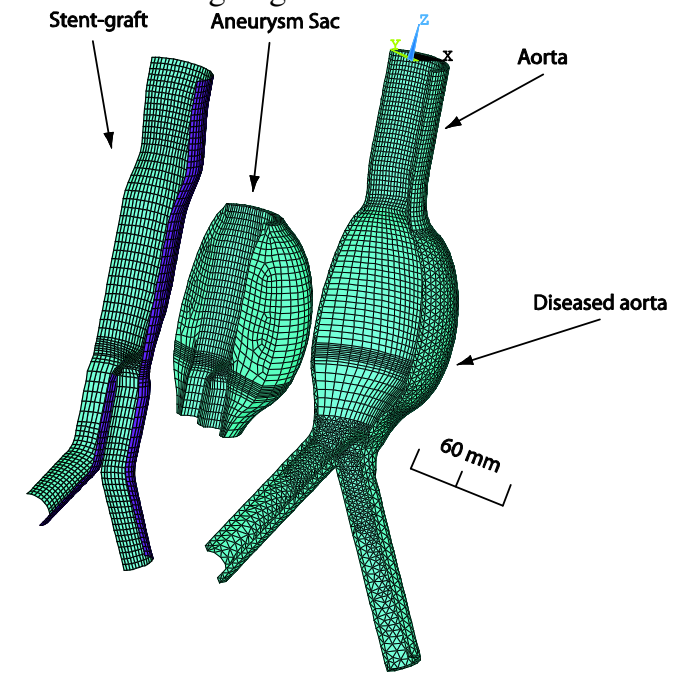

Fig. 6. Mechanical model mesh.

The mechanical domain of the simulation assumes zero displacement at the top of the AAA neck and at the bottom of the iliacs, while a time dependent uniform velocity is applied at the inlet of the fluidic domain (Fig. 7a) and a time dependent normal traction (due to luminal pressure) on the outlet (Fig. 7b). The transient analysis is performed during a full cardiac cycle $(1.1 \mathrm{~s})$. 

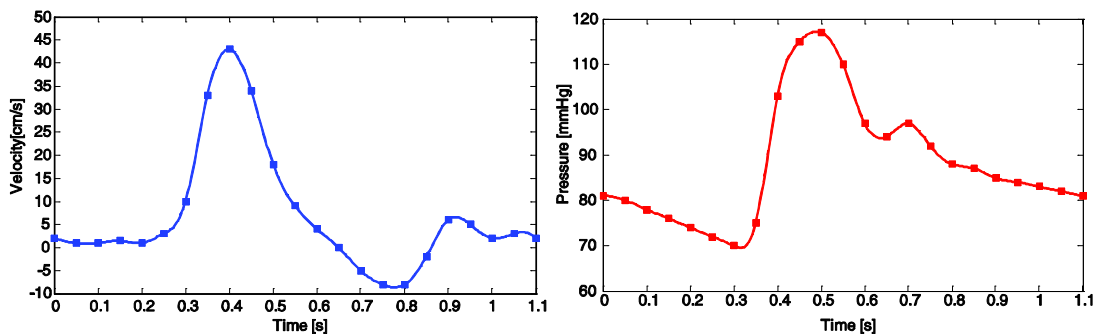

Fig. 7. Fluidic boundary conditions: a) inlet velocity and b) outlet pressure.

To validate the FSI solver, simulations using a simpler AAA model without stent-graft were performed. The results showed a maximum aorta displacement of $1.9 \mathrm{~mm}$ and a maximum stress (von Mises) around $0.4 \mathrm{MPa}$, which were in good agreement with the data from [20] (using a similar geometry size).

Simulation results of the pressure distribution within the aneurysm sac at systolic pressure $(\mathrm{t}=0.5 \mathrm{~s})$ using the full AAA model with stent-graft are shown in Fig. 8a. The simulation results show some small pressure variations along the aneurysm sac, with the minimum pressure occurring close to the stent-graft bifurcation. A closer look to the stent-graft displacement at the same simulation time (Fig. 8b) suggests that the minimum pressures are related to the maximum stentgraft displacements.

a)

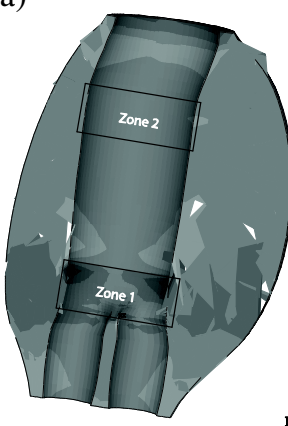

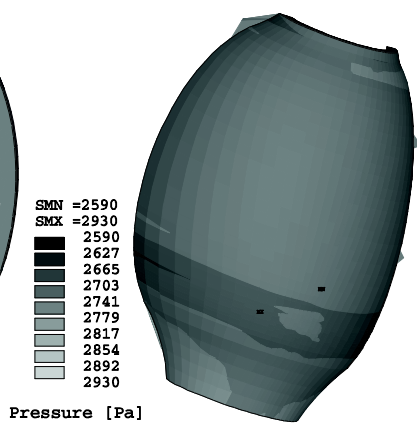

b)

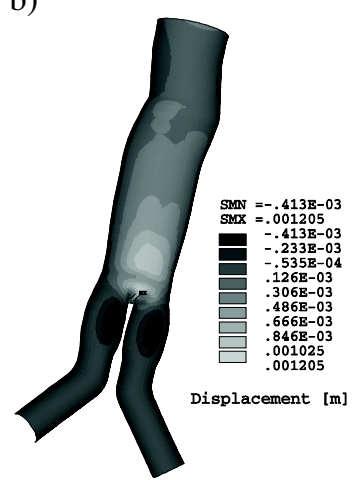

Fig. 8. Simulation results at systolic pressure $(t=0.5 \mathrm{~s})$. a) aneurysm sac pressure and $\mathrm{b}$ ) stentgraft displacement.

The results from Fig. 8a show that the pressure within the aneurysm sac is almost uniform but do not give any information about the aneurysm sac pressure variation during a full cardiac cycle. Therefore, if two zones are defined (zone 1 and 2 in Fig. 8a) the mean pressures within those regions can be computed. The mean pressures in the two zones along with the pressure boundary condition at the outlet are depicted in Fig. 9. 


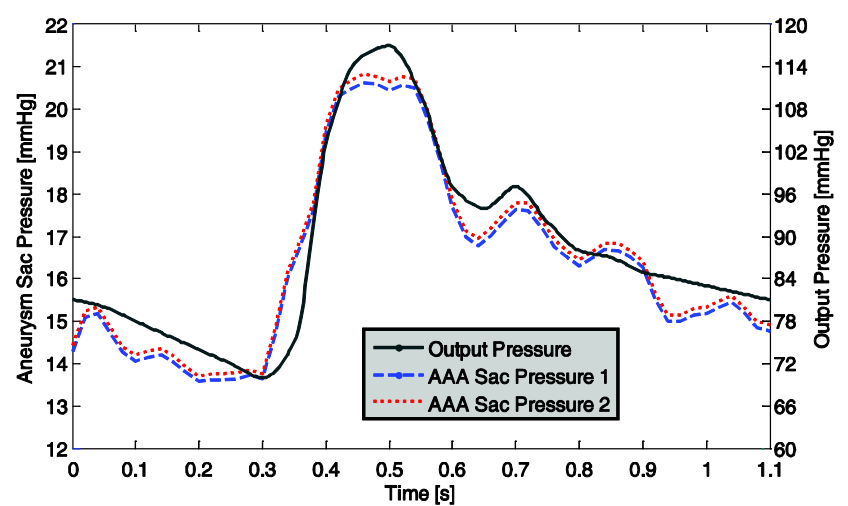

Fig. 9. Pressures inside aneurysm sac (zone 1 and 2 in Fig. 8a) and pressure boundary condition at the outlet.

These simulation results imply that the pressure variations within the aneurysm sac are related to the displacement of the stent-graft caused by the luminal pressure. If this is the case, the placement of one sensor on the region with less structural stability (higher displacement) might be a good indicator, when compared to another sensor placed elsewhere within the aneurysm sac, of the structural integrity of the stent-graft. These results also suggest that the pressure variations inside the aneurysm sac are related to the stent-graft material (structural behavior). Results for the same simulation boundary conditions but using a higher Young's Modulus for the stent-graft material, $\mathrm{E}=60 \mathrm{MPa}$, Fig. 10, confirm the assumption that the pressure within the aneurysm sac depends on the structural behavior of the stent-graft material. In fact, simulations reveal that a large drop on the pressure within the aneurysm (> $10 \mathrm{mmHg}$ ) sac occurs when the Young's Modulus is increased from $10 \mathrm{MPa}$ to $60 \mathrm{MPa}$.

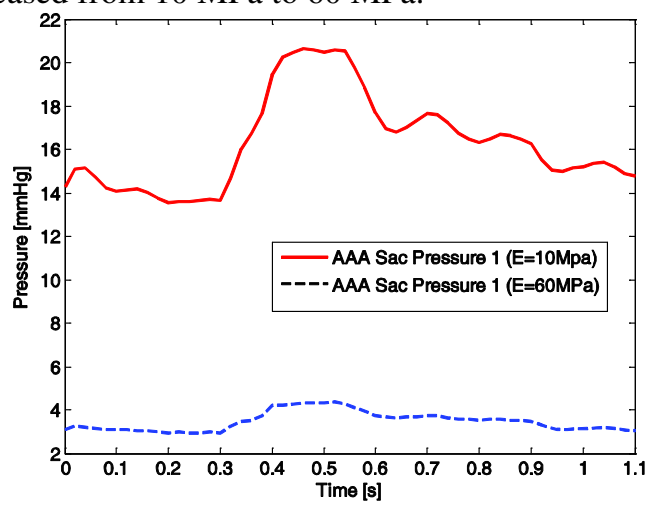

Fig. 10. Pressures inside aneurysm sac on zone 1 (see Fig. 8a) for different stent-graft material properties.

The abdominal aortic aneurysm CFD (Compute Fluid Dynamics) model with FSI is a suitable tool to study pressure changes within the aneurysm and indicates 
that pressure sensors can be used to detect post-EVAR complications. Pressure sensing in the aneurysm sac can be used both for leakage detection and to measure systolic and diastole blood pressures. If more than one sensor is used, model results suggest that the differences in pressure within the aneurysm sac can be an indicator of the stent-graft material integrity.

\section{Development of a Flexible Pressure Sensor}

A key component for the smart stent-graft is the pressure sensor. The sensing element must be flexible to enable its conformability to the stent-graft and thus the aorta. Such features allow the attachment of the sensor to the stent-graft and their deployment in a single step (as opposed to the requirement of two catheters as in the EndoSure device). Furthermore, it enables the placement of more than one sensor (a sensor cluster) contributing to a more comprehensive study of postEVAR aneurysm evolution that, currently, is not possible.

\section{Capacitive sensor design}

Research on implantable pressure sensors is very active and has been supported and justified by the need of continuous pressure monitoring for patients with congestive heart failure, as an early diagnostic mechanism for some risk patients and for post-EVAR surveillance [24-25].

Implantable pressure sensors are typically categorized into extra-arterial blood pressure devices and intra-arterial blood pressure devices [25]. The firsts are placed around the blood vessel and perform an indirect pressure measurement through the wall or through the expansion and contraction of the artery. They require an invasive surgical procedure for their implant while, on the other hand, the intra-arterial devices are in contact with the blood stream inside of the blood vessels.

After stent-graft placement, the aneurysm sac gets depressurized and the pressure drops down to a few $\mathrm{mmHg}$ as indicated by the simulations $(12-22 \mathrm{mmHg}$ according to Fig. 9). Therefore, if one wants to sense the luminal pressure value (ranges typically between $50-160 \mathrm{mmHg}$ ) through the aneurysm sac pressure, the sensor must be able to measure pressures between $6-26 \mathrm{mmHg}$. In addition, it needs a high dynamic range in order to detect stent-graft complications (in this case the sac gets pressurized and pressure increases to the luminal pressure values).

Typical configurations of capacitive pressure sensors use square-plate (diaphragm) electrodes separated by a dielectric (oftentimes of air) at a pressure $\mathrm{P}_{0}$. Changes on the outside pressure ( $\left.\mathrm{P}_{\mathrm{out}}\right)$ deform the square plate and consequently generate a capacitive change. A schematic of a square-plate (side length of $2 \mathrm{a}$ ) 
pressure sensor is shown in Fig. 11. The sensor involves two coupled domains, mechanical and electrical, that define the sensor behavior.

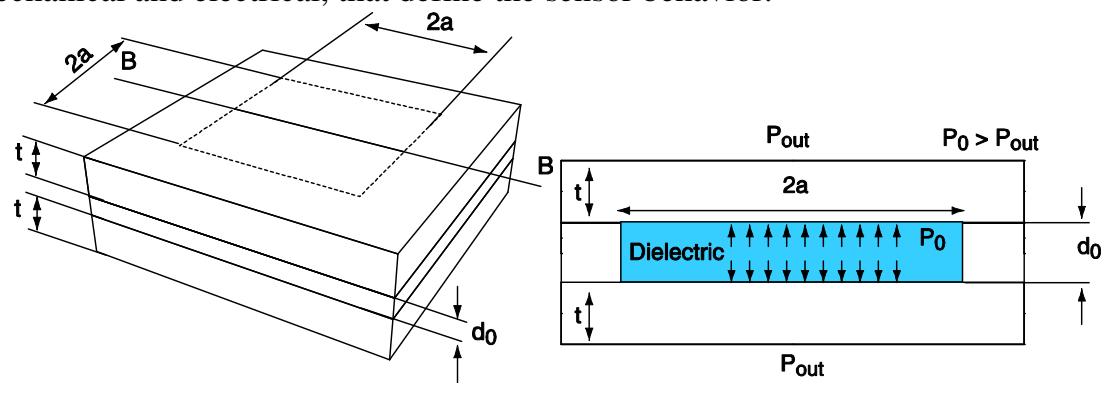

Fig. 11. Schematic of the pressure square (side length $=2 a$ ) sensor a) $3 \mathrm{D}$ view and $b$ ) section cut B-B.

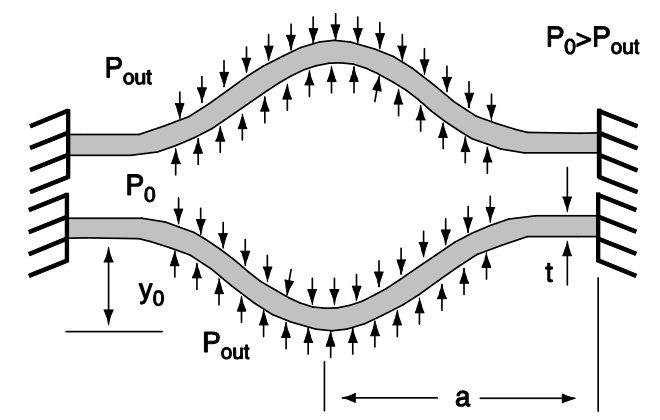

Fig. 12. Cross section of a generic deflectable diaphragm.

A cross section of the square plate sensor is shown in Fig. 12 (section cut B-B) where only the mechanical domain is considered. The side length is $2 \mathrm{a}, \mathrm{t}$ is the thickness and yo the deflection. The diaphragm is clamped at the edges. For a clamped diaphragm under a uniform load (like pressure), the angle of deflection, $\varphi$, is equal to zero at the center $(r=0)$ and at the edge $(r=a)$ of the diaphragm. For these boundary conditions, the deflection of an isotropic square diaphragm under a pressure load can be modeled as [26]:

$$
P_{0}-P_{\text {out }}=\frac{E t^{4}}{\left(1-v^{2}\right) a^{4}}\left[4.20 \frac{y_{0}}{t}+1.58 \frac{y_{0}{ }^{3}}{t^{3}}\right]
$$

where $v$ is the Poisson's ratio, $E$ is the Young's modulus, and $\Delta P=P_{0}-P_{\text {out }}$ is the pressure load.

Equation (1) allows the calculation of the deflection at the center of the diaphragm for a given pressure load but the deflection along the diaphragm is still required to model the capacitive changes due to gap variation. Due to the complexity of the mechanical deflection calculation, trial functions that describe the deflection of the entire diaphragm are usually used [27]. Fig. 13 shows the norma- 
lized deflection for a square diaphragm of side-length $2 \mathrm{a}$ when the following trial function for large deflection is used [27]:

$$
y(x, y)=y_{0}\left[\left(\cos \left(\frac{\pi x}{2 a}\right)\right)\left(\cos \left(\frac{\pi y}{2 a}\right)\right)\right]
$$

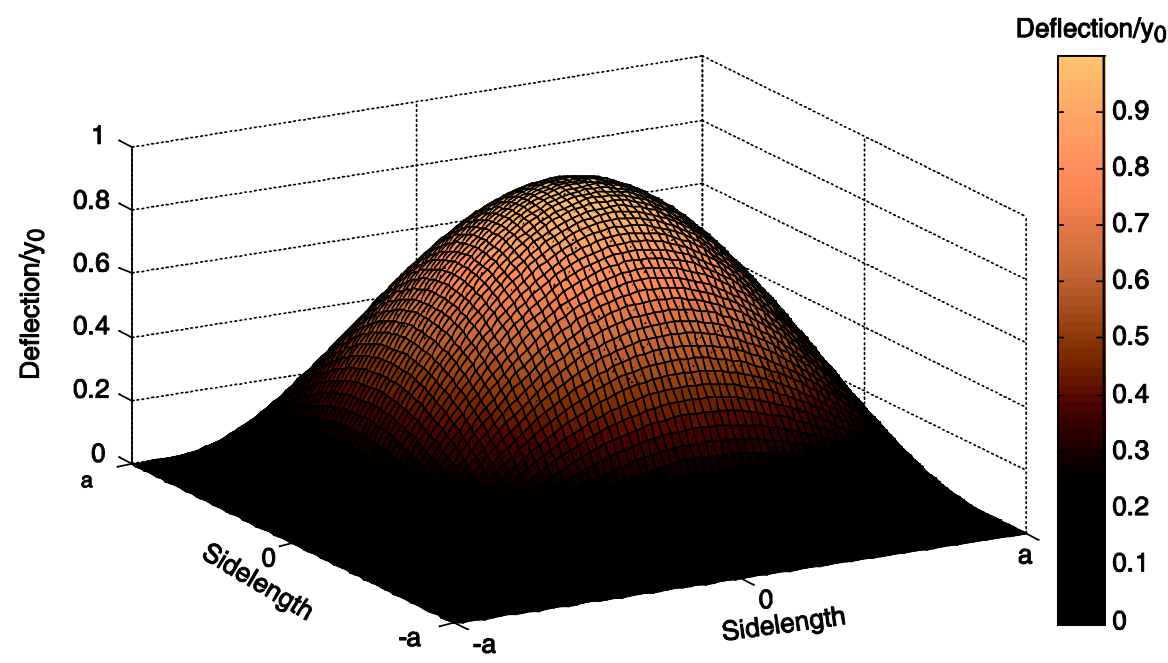

Fig. 13. Displacement profile along the square diaphragm using the trial function of equation (2).

Mechanical deflections caused by pressure changes will originate changes at the capacitor (electrostatic domain). A capacitor is an electronic component with two electrodes that are separated by a dielectric. For the simple case of a parallel plate capacitor, and in the absence of displacements, the model for the capacitor is (neglecting fringe fields):

$$
C=\varepsilon_{0} \varepsilon_{r} \frac{w l}{d_{0}}
$$

where $\varepsilon_{0}$ is the permittivity of free space $(8.8546 \times 10-12 \mathrm{~F} / \mathrm{m}), \mathcal{E}_{r}$ is the relative permittivity, $w$ and $l$ are the width and length of the capacitor electrodes, and $d_{0}$ is the gap between the electrodes.

Since the capacitive sensor uses diaphragm electrodes with a complex bending profile, integration over the effective area of the electrodes is required to compute the total capacitance:

$$
C=\iint \frac{\varepsilon_{0} \varepsilon_{r}}{d_{0}+2 y(x, y)} d x d y
$$


where $\mathrm{y}(\mathrm{x}, \mathrm{y})$ is the distance between electrodes due to the diaphragm bending at position $\mathrm{x}, \mathrm{y}$. The integration in equation (4) can easily be solved numerically, enabling the computation of the capacitance for a given pressure change.

\section{Fabrication process}

Given the characteristics of the application (the sensor will be attached to the stent-graft) the capacitive sensor must be foldable, extremely flexible and characterized by a very small profile. In addition, the technology should be simple and biocompatible. Silicon based micro technologies are widely used in implantable medical devices [24], but due to the application specifications, there are other technologies that can deliver better design approaches and results.

A suitable candidate is the technology proposed by Sepúlveda [28], in which aligned carbon nanotubes (CNTs) are used to implement the conductive elements in a flexible substrate of polydimethylsiloxane (PDMS), a transparent, nontoxic and biocompatible silicone elastomer.

The technology process flow enabling the fabrication of a flexible pressure sensor is schematically presented in Fig. 14. Acrylic moulds are produced by CNC milling (Fig. 14a) for posterior fabrication of the PDMS membranes. This technique has low costs and fast production times, but it is associated with poor dimensional control (dimensions less than $50 \mu \mathrm{m}$ are difficult to achieve) and more traditional micromachining may achieve the required tolerances.

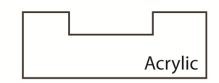

Mold Fabrication
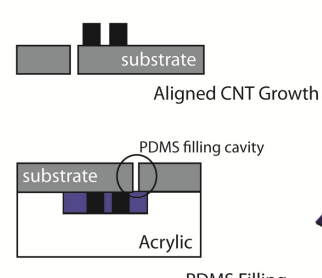
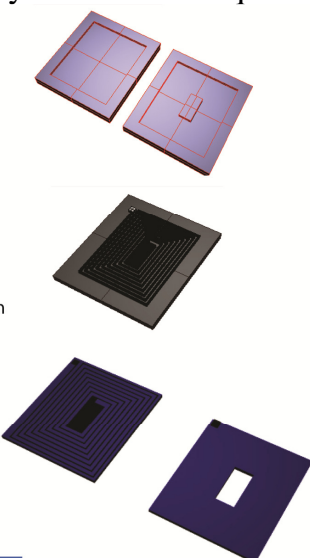

$\square$ CNT $\square$ PDMS

PDMS Bonding

Fig. 14. Fabrication process flow for the development of a flexible pressure sensor with alignedCNT/PDMS nanocomposites.

The electrical components are based on aligned CNTs, as shown in Fig. 14b. Chemical vapor deposition (CVD) is used to grow forests or "carpets" of vertical- 
ly-aligned CNTs [29]. A silicon substrate with patterned $\mathrm{Fe} / \mathrm{Al}_{2} \mathrm{O}_{3}$ catalyst is placed on a horizontal quartz tube furnace at atmospheric pressure at $750{ }^{\circ} \mathrm{C}$ [29] for the CNT growth. This method has the advantage of allowing the growth of high purity, high yield and vertically aligned CNTs. Next, the CNTs are embedded into the polymer matrix (PDMS). This step is schematically represented in Fig. 14c. The substrate is placed against the moulds, and the PDMS is introduced in the cavities through a hole to create an aligned-CNT/PDMS nanocomposite as described previously for epoxies using capillarity-assisted wetting [30], followed by the curing of the elastomer.

Three flexible membrane layers are required to fabricate the sensor, with the top and bottom layers defining the inductor and the electrodes, and the middle one defining the dielectric (air). This configuration requires bonding of PDMS membranes. Eddings [31] tested five different bonding techniques and the highest reported bond strength was obtained for both partial curing and uncured PDMS adhesive techniques. The latter approach is used to build the sensors, Fig. 15.

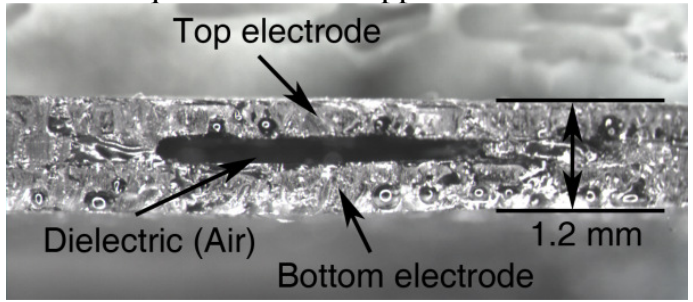

Fig. 15. Cross section of a CNT/PDMS flexible pressure sensor.

\section{Material properties and results}

The key step of the fabrication process is the CNT-PDMS impregnation and respective mechanical and electrical properties (that will govern the sensor response). Aligned CNTs are oriented in the out-of-plane (or normal to the wafer plane) direction such that the polymer nanocomposite can be presumed transversly isotropic, i.e., isotropic in the plane of the sensor. Furthermore, the modulus enhancement due to CNTs is likely minimal as the long axis of the CNTs are oriented perpendicular to the loading direction, such that the PDMS polymer dominates the response. Work on nanocomposites has shown significant increase in modulus due to aligned CNTs in polymer (PDMS) [32] and epoxy [33] in the CNT axis direction, but little reinforcement effect in the transverse direction as used here. This result is expected from composite micromechanics analyses and experimental results.

Experimental results using a prototype PDMS/CNT flexible pressure sensor are presented in Fig. 16. The tested sensors have a mechanical layer thickness of 670 $\mu \mathrm{m}$ and a dielectric thickness of $260 \mu \mathrm{m}$ resulting in a total sensor thickness of $1.6 \mathrm{~mm}(670 \mu \mathrm{m}+260 \mu \mathrm{m}+670 \mu \mathrm{m})$. The area of the electrodes is $3.4 \times 9 \mathrm{~mm}^{2}(\mathrm{~W}$ 
$x$ L). Despite the coarse sensor resolution (the geometry was not optimized since the devices are at a proof-of-concept stage), capacitive changes were measured when the sensor was placed inside a controlled pressure chamber (the dielectric is hermetically sealed at ambient pressure).

a)

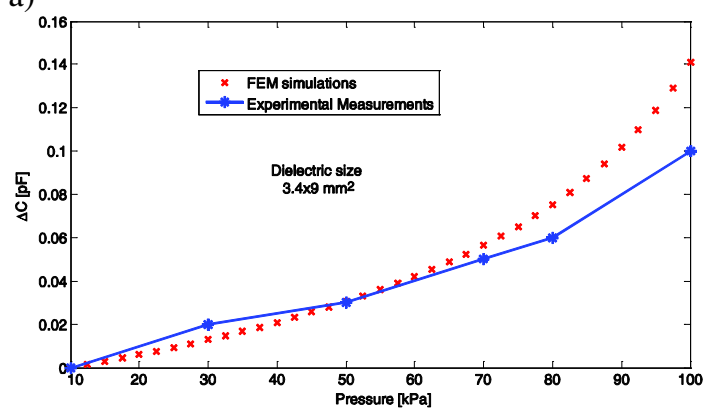

b)

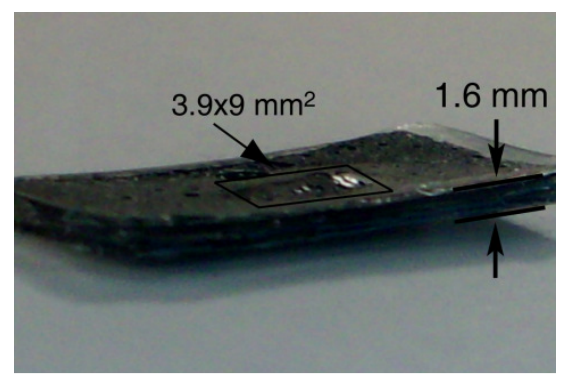

Fig. 16. Experimental results of a prototype CNT/PDMS flexible pressure sensor for a smart stent-graft. a) Capacitive changes vs. pressure and b) image of the sensor with $10 \mathrm{kPa}$ external pressure.

\section{Conclusions}

Since its introduction, in the beginning of the 90's, EVAR became a viable alternative for the treatment of aneurysms. Despite the benefits of being a minimally invasive technique, recent cost-benefit analyses indicate that EVAR becomes more expensive on the long-term since it requires surveillance mechanisms to detect eventual post-EVAR problems, such as endoleaks and stent-graft migration.

Alternatives are currently being pursued to minimize the surveillance costs, and pressure measurements in the aneurysm sac are an attractive solution. Some stand alone pressure sensors are already available but the future will evolve to smart stent-grafts, i.e., stent-grafts with embedded pressure sensors for post-EVAR surveillance.

The use of pressure changes as a surveillance mechanism still requires a better understanding from the medical community. Nevertheless, AAA model simulation 
results validate the use of pressure sensors to detect post-EVAR complications. Although more data is required, multiple pressure measurements within the aneurysm sac can also enable the detection of stent-graft structural failure.

In order to place the sensors in the stent-graft, they must be thin and highly flexible. A newly developed technology based on CNTs has proven successful in the development of flexible pressure sensors and might enable the next generation of smart stent-grafts.

\section{Acknowledgments}

The first and second authors wishes to thank FCT - Fundação para a Ciência e Tecnologia in Portugal, for the financial support provided by the $\mathrm{PhD}$ grants with reference SFRH/BD/42967/2008 and SFRH/BD/42922/2008 respectively.

This work is supported by FCT under the project MIT-Pt/EDAMEMD/0007/2008 (http://sensecardiohealth.wordpress.com).

The authors wish to thank Miguel Marafuz for the illustrations (http://illustrationmfz.wordpress.com/), Fabio Fachin for helpful discussions in the CNT-based MEMS device designs, and Dr. Roberto Guzman de Villoria for fabrication and testing assistance of the CNT-reinforced PDMS materials.

CNT-based polymer composite materials were developed with funding from Airbus S. A. S., Boeing, Embraer, Lockheed Martin, Saab AB, Spirit AeroSystems, Textron Inc., Composite Systems Technology, and TohoTenax Inc. through MIT's Nano-Engineered Composite aerospace STructures (NECST) Consortium.

\section{References}

1. Johnston KW, Robert BR, Tilson MD, Dhiraj MS, Larry H, James CS (1991) Suggested standards for reporting on arterial aneurysms. Journal of Vascular Surgery 13 (3):452-458

2. Ricotta II JJ, Malgor RD, Oderich GS (2009) Endovascular abdominal aortic aneurysm repair: Part I. Annals of Vascular Surgery 23 (6):799-812. doi:10.1016/j.avsg.2009.03.003

3. Katzen BT, MacLean AA (2006) Complications of endovascular repair of abdominal aortic aneurysms: A review. CardioVascular and Interventional Radiology 29 (6):935-946. doi:10.1007/s00270-005-0191-0

4. Baril DT, Kahn RA, Ellozy SH, Carroccio A, Marin ML (2007) Endovascular abdominal aortic aneurysm repair: Emerging developments and anesthetic considerations. Journal of Cardiothoracic and Vascular Anesthesia 21 (5):730-742. doi:10.1053/j.jvca.2007.03.001

5. Young KC, Awad NA, Johansson M, Gillespie D, Singh MJ, Illig KA (2010) Cost-effectiveness of abdominal aortic aneurysm repair based on aneurysm size. Journal of Vascular Surgery 51 (1):27-32. doi:10.1016/j.jvs.2009.08.004

6. Myers K, Devine T, Barras C, Self G (2001) Endoluminal versus open repair for abdominal aortic aneurysms. Paper presented at the 2do. Congreso Virtual de Cardiología - Congreso Internacional de Cardiología por Internet, Argentina, Set 1 - Nov 30, 2001

7. Dion Y-M, Joseph T (2010) Laparoscopic aortic surgery. In: Fogarty TJ, White RA (eds) Peripheral Endovascular Interventions. Springer New York, pp 397-409. doi:10.1007/978-1-4419-1387-6_27 
8. Greenhalgh RM (2004) Comparison of endovascular aneurysm repair with open repair in patients with abdominal aortic aneurysm (EVAR trial 1), 30-day operative mortality results: randomised controlled trial. The Lancet 364 (9437):843-848. doi:10.1016/S0140-6736(04)16979-1

9. Milner R, Kasirajan K, Chaikof EL (2006) Future of endograft surveillance. Seminars in Vascular Surgery 19 (2):75-82. doi:10.1053/j.semvascsurg.2006.03.002

10. Gilling-Smith G, Brennan J, Harris P, Bakran A, Gould D, McWilliams R (1999) Endotension after endovascular aneurysm repair: definition, classification, and strategies for surveillance and intervention. Journal of Endovascular Surgery 6:305-307

11. Carnero L, Milner R (2007) Aneurysm sac pressure measurement with a pressure sensor in endovascular aortic aneurysm repair. In: Alan B. Lumsden, Lin PH, Chen C, Parodi JC (eds) Advanced endovascular therapy of aortic disease. pp 209-215. doi:10.1002/9780470988930.ch27

12. Ellozy SH, Carroccio A, Lookstein RA, Minor ME, Sheahan CM, Juta J, Cha A, Valenzuela R, Addis MD, Jacobs TS, Teodorescu VJ, Marin ML (2004) First experience in human beings with a permanently implantable intrasac pressure transducer for monitoring endovascular repair of abdominal aortic aneurysms. Journal of Vascular Surgery 40 (3):405-412. doi:10.1016/j.jvs.2004.06.027

13. Schlierf R, Gortz M, Schmitz Rode T, Mokwa W, Schnakenberg U, Trieu K Pressure sensor capsule to control the treatment of abdominal aorta aneurisms. In: Solid-State Sensors, Actuators and Microsystems, 2005. Digest of Technical Papers. TRANSDUCERS '05. The 13th International Conference on, 2005. pp 1656-1659 Vol. 1652. doi:10.1109/SENSOR.2005.1497407

14. Springer F, Schlierf R, Pfeffer J-G, Mahnken A, Schnakenberg U, Schmitz-Rode T (2007) Detecting endoleaks after endovascular AAA repair with a minimally invasive, implantable, telemetric pressure sensor: an in vitro study. European Radiology 17 (10):2589-2597. doi:10.1007/s00330-0070583-4

15. Springer F, Günther R, Schmitz-Rode T (2008) Aneurysm sac pressure measurement with minimally invasive implantable pressure sensors: An alternative to current surveillance regimes after EVAR? CardioVascular and Interventional Radiology 31 (3):460-467. doi:10.1007/s00270-007-9245-9 16. Rutherford RB, Krupski WC (2004) Current status of open versus endovascular stent-graft repair of abdominal aortic aneurysm. Journal of Vascular Surgery 39 (5):1129-1139. doi:10.1016/j.jvs.2004.02.027

17. Hayter CL, Bradshaw SR, Allen RJ, Guduguntla M, Hardman DTA (2005) Follow-up costs increase the cost disparity between endovascular and open abdominal aortic aneurysm repair. Journal of Vascular Surgery 42 (5):912-918. doi:10.1016/j.jvs.2005.07.039

18. Kuribayashi K, Tsuchiya K, You Z, Tomus D, Umemoto M, Ito T, Sasaki M (2006) Selfdeployable origami stent grafts as a biomedical application of Ni-rich TiNi shape memory alloy foil. Materials Science and Engineering: A 419 (1-2):131-137. doi:10.1016/j.msea.2005.12.016

19. Rigberg D, Tulloch A, Chun Y, Mohanchandra KP, Carman G, Lawrence P (2009) Thin-film nitinol (NiTi): A feasibility study for a novel aortic stent graft material. Journal of vascular surgery : official publication, the Society for Vascular Surgery [and] International Society for Cardiovascular Surgery, North American Chapter 50 (2):375-380. doi:10.1016/j.jvs.2009.03.028

20. Li Z, Kleinstreuer C (2006) Analysis of biomechanical factors affecting stent-graft migration in an abdominal aortic aneurysm model. Journal of Biomechanics 39 (12):2264-2273. doi:10.1016/j.jbiomech.2005.07.010

21. Frauenfelder T, Lotfey M, Boehm T, Wildermuth S (2006) Computational fluid dynamics: Hemodynamic changes in abdominal aortic aneurysm after stent-graft implantation. CardioVascular and Interventional Radiology 29 (4):724-724. doi:10.1007/s00270-005-8227-z 
22. Scotti CM, Finol EA Compliant biomechanics of abdominal aortic aneurysms: A fluid-structure $\begin{array}{llllll}\text { interaction study. } & \text { Computers } \quad \& \quad \text { Structures } & 85 & \text { (11-14):1097-1113. }\end{array}$ doi:10.1016/j.compstruc.2006.08.041

23. Vorp DA (2007) Biomechanics of abdominal aortic aneurysm. Journal of Biomechanics 40 (9):1887-1902. doi:DOI: 10.1016/j.jbiomech.2006.09.003

24. Rogier AMR, et al. (2007) Microsystem technologies for implantable applications. Journal of Micromechanics and Microengineering 17 (5):R50. doi:10.1088/0960-1317/17/5/R02

25. Potkay J (2008) Long term, implantable blood pressure monitoring systems. Biomedical Microdevices 10 (3):379-392. doi:10.1007/s10544-007-9146-3

26. Chau H, Wise K (2005) Scaling limits in batch-fabricated silicon pressure sensors. Electron Devices, IEEE Transactions on 34 (4):850-858. doi:10.1109/T-ED.1987.23006

27. Senturia SD (2000) Microsystem design. Springer,

28. Sepúlveda AT, Moreira A, Fachin F, Wardle BL, Silva JM, Pontes AJ, Viana JC, Rocha LA Inductive-coupling system for abdominal aortic aneurysms monitoring based on pressure sensing. In: MME'2010 (21th MicroMechanics Europe), Enschede, The Netherlands, September 26-29, 20102010. 29. Hart AJ, Slocum AH (2006) Rapid growth and flow-mediated nucleation of millimeter-scale aligned carbon nanotube structures from a thin-film catalyst. The Journal of Physical Chemistry B 110 (16):8250-8257. doi:10.1021/jp055498b

30. Wardle BL, Saito DS, García EJ, Hart AJ, de Villoria RG, Verploegen EA (2008) Fabrication and characterization of ultrahigh-volume-fraction aligned carbon nanotube-polymer composites. Advanced Materials 20 (14):2707-2714. doi:10.1002/adma.200800295

31. Eddings MA, Johnson MA, Gale BK (2008) Determining the optimal PDMS-PDMS bonding technique for microfluidic devices. Journal of Micromechanics and Microengineering 18 (6):067001. doi:10.1088/0960-1317/18/6/067001

32. Ajayan PM, Schadler LS, Giannaris C, Rubio A (2000) Single-walled carbon nanotube-polymer composites: Strength and weakness. Advanced Materials 12 (10):750-753. doi:10.1002/(sici)15214095(200005)12:10<750::aid-adma750>3.0.co;2-6

33. Cebeci H, Villoria RGd, Hart AJ, Wardle BL (2009) Multifunctional properties of high volume fraction aligned carbon nanotube polymer composites with controlled morphology. Composites Science and Technology 69 (15-16):2649-2656. doi:10.1016/j.compscitech.2009.08.006 